\title{
The Use of Statistical Functions for the Selection of Laser Texturing Parameters
}

https://doi.org/10.1515/eng-2020-0044

Received Dec 16, 2019; accepted Apr 08, 2020

\begin{abstract}
Laser surface texturing is currently the most developed technique for producing fully reproducible microcavities on the surfaces of machine elements. From the point of view of texture technology, an important aspect is the proper selection of process parameters to obtain texture elements with desirable and repetitive geometries and physicochemical properties. Surface texturing improves mottling and fretting resistance and is also used wherever the adhesion properties of surface layers (printing techniques, bonding materials, biological and chemical activity, coatings, etc.) are important. The article shows the possibility of applying statistical functions to the selection of appropriate machining parameters to obtain microgeometry useful in the application of textured surfaces [1].
\end{abstract}

Keywords: Laser Surface Texturing, LST

\section{Introduction}

The development of technology places very high demands on the reliability and durability of machine elements, while constantly increasing the threshold values for their operating parameters. One of the ways to meet these demands is to direct the efforts of designers and technologists to activities towards involving the formation of microgeometry of selected surfaces of machine elements. This is facilitated by advances in technology, which in recent years has made good progress, especially in the area of laser technology, enabling local intensified impact on the workpiece.

In order to achieve maximum material utilization and the best possible material performance, it is extremely important to modify the material surface properties. For example, it is possible to influence the surface morphology

\footnotetext{
*Corresponding Author: Piotr Sęk: Politechnika Świętokrzyska w Kielcach, Centrum Laserowych Technologii Metali (Kielce University of Technology, Centre for Laser Technologies of Metals), Al. 1000lecia P. P. 7, PL- 25-314 Kielce
}

๑ Open Access. ๑ 2020 P. Sęk, published by De Gruyter. and its absorption properties by changing the structure of the material [2] or chemical impurities contained in the surface. The susceptibility of a material to abrasion and surface damage may be reduced by changing the chemical composition, morphology and crystal structure of its surface [3]. Moreover, it can beassumed that the forces of friction, adhesion and wetting occurring in the transitional (interphase) layer of the material are largely determined by the size and shape of the elements in the micro and nanoscale [4]. Currently, surface engineering focuses on the modification of surface microgeometry in order to improve its performance properties, while trying not to affect the structure and mechanical properties of the workpiece.

A laser beam is a carrier that allows to obtain a very high concentration of energy in terms of area and time of impact on the workpiece. In laser micromachining, the area of the actual impact on the material is determined by the size of the focused laser dot or the mask that transmits only the desired portion of the beam. The impact of the beam pulse time is particularly important as different mechanisms of impact on the material can be used depending on the radiation intensity and exposure time. This is due to the finite response times of electrons and atomic network of the material to photons. One of the main advantages of laser as a tool for material processingis the ability to precisely control where and at what rate energy is deposited. This control is carried out by appropriate selection of laser processing parameters in order to achieve the desired material modification [1,5-8].

Laser ablation is the removal of material from the substrate by direct absorption of laser energy. Laser ablation is usually discussed in connection with pulse lasers, but it is also possible in the case of intense radiation of permanent waves. Above the ablation threshold, the thickness or volume of the material ablated per pulse usually exhibits a logarithmic increase together with fluence for short pulses or a linear increase for long pulses according to Beer-Lambert's law. Microsecond and nanosecond pulses are long pulses whereas picosecond and femtosecond pulses are short or ultrashort pulses [1].

During laser ablation, different material removal mechanisms can activate depending on the specific mate- 
rial layout and laser processing parameters such as wavelength, fluence and pulse length $[9,10]$. At low fluences, photothermal ablation mechanisms include evaporation and sublimation of a material. In the case of multicomponent systems, more volatile components can be depleted more quickly, changing the chemical composition of the remaining material. At higher fluences, the heterogeneous nucleation of steam bubbles leads to normal boiling.

The laser pulse length can have a significant impact on the dynamics of the ablation process. In general, since the pulse duration is shortened, energy is stored more quickly in the material, which leads to its faster removal. The amount of material excited directly by the laser has less time to transfer energy to the surrounding material before ablation [9].

Surface texturing has been used for many years as a method of improving tribological properties of surfaces. The formation of a controlled texture on one of two sliding surfaces in relative motion can have many positive effects, such as reduction of friction and wear and increase in the capacity of a sliding pair $[1,5]$.

In industrial processes such as protective coating application, catalysis, lubrication, flotation, gluing and printing, wettability of solids with various liquids is extremely important in practice. Studies of the properties of the surface layer of various solids, including surface free energy and wettability, which constitute important elements of the assessment of adhesive properties of solids necessary for the analysis of the effects of modification of their surface layer, have been underway for many years. Wettability and the SEP have long had its place in the research in the field of physics, chemistry, biology, materials engineering, and most recently in surface engineering [11].

Laser surface texturing surpasses mechanical (e.g. shot blasting, honing or blasting), chemical and electrical (electrical discharge texturing) methods because it allows local changes with a high degree of control over the shape and size of the resulting components and allows for greater size variation. It is generally cheaper than electron beam texturing and is therefore less restricted because it does not require a vacuum. Various textures can be produced in a well-defined way by controlling process parameters such as beam intensity, spatial and time profile, wavelengths and selection of the environment (gaseous or liquid). The basic dimensions of surface attributes (e.g. the width of a melted or ablation area) are usually determined by the shape and size of the beam. In the case of LST, however, secondary attributes may form around the radiation area at microscale or even nanoscale due to the variety of mechanisms involved, including melting after ablation, re-solidification, or surface liquid splashes resulting from the jet pressure. These secondary attributes may be as important as the primary dimensions when determining the function of the material in the selected application $[5,12$ 15].

Currently, surface engineering focuses on the modification of surface microgeometry in order to improve its performance properties, while trying not to affect the structure and mechanical properties of the workpiece.

Currently, many studies are available on the impact of LST application on the operational properties of machine components. Most of them focus on the impact of texture application on friction reduction in sliding friction pairs and adjusting adhesive properties of modified surfaces. Yet mostly it is associated with the mere application of this solution. In my previous research $[1,5,12]$ I showed that the most important factor influencing the operational behavior of a textured surface is the microgeometry of individual texture elements. The most important factor affecting the reduction of friction is the ratio of depth to diameter of the texture element and in the case of impact on contact angles and free surface energy, the most important microgeometry parameter is the mutual distance of the texture elements. It is essential to study the influence of machining parameters on texture geometry and to use of appropriate parameters for obtaining a texture with geometry that will have a significant impact on the planned application.

\section{Experiment}

When producing the test samples, the average power range of laser radiation from $5 \mathrm{~W}$ to $1.5 \mathrm{~W}$ and laser pulse frequencies of $400 \mathrm{kHz}, 200 \mathrm{kHz}, 133.33 \mathrm{kHz}, 100 \mathrm{kHz}, 80 \mathrm{kHz}$ and $66.66 \mathrm{kHz}$ were examined. For average power less than $1.5 \mathrm{~W}$ and frequencies less than $66.66 \mathrm{kHz}$, the fluence was less than $1.33 \mathrm{~J} / \mathrm{cm}^{2}$; no significant influence of machining on the material or ablative mass loss was observed. It can therefore be concluded that $1.33 \mathrm{~J} / \mathrm{cm}^{2}$ is the threshold fluence $n$ for $100 \mathrm{Cr} 6$ steel. All samples were produced at a constant scanning speed of $100 \mathrm{~mm} / \mathrm{s}$. The set diameter of the microcavity was $100 \mu \mathrm{m}$. The surfaces of the samples were prepared before laser processing on a specimen polisher ( $\mathrm{Ra}=0.392 \div 0.694, \mathrm{Rz}=1.336 \div 1.919)$.

Taylor Hobson's Talysurf CCI optical profiler, using a consistent correlation interferometry method with a range of $2.2 \mathrm{~mm}$ and a resolution of $0.01 \mathrm{~nm}$ in an $\mathrm{x} 50$ zoom lens to measure $330 \mu \mathrm{m} \times 330 \mu \mathrm{m} \times 27.7^{\circ}$ slope and $330 \mu \mathrm{m}$ area, was used to determine the geometry of the microcavities. For each sample, 3 measurements were taken concentrating on the depth of the microcavity, its volume, the height 
Table 1: Comparison of parameters of laser micromachining of tested samples

\begin{tabular}{|c|c|c|c|c|c|c|c|}
\hline $\mathrm{N}$ & $\mathrm{P}[\mathrm{W}]$ & $\mathrm{f}[\mathrm{kHz}]$ & $\mathrm{F}\left[\mathrm{J} / \mathrm{cm}^{2}\right]$ & $\mathrm{N}$ & $\mathrm{P}[\mathrm{W}]$ & $\mathrm{f}[\mathrm{kHz}]$ & $\mathrm{F}\left[\mathrm{J} / \mathrm{cm}^{2}\right]$ \\
\hline 1 & 5 & 400 & 4.76 & 46 & 3.25 & 100 & 2.96 \\
\hline 2 & 5 & 200 & 4.69 & 47 & 3.25 & 80 & 2.92 \\
\hline 3 & 5 & 133.3 & 4.62 & 48 & 3.25 & 66.66 & 2.88 \\
\hline 4 & 5 & 100 & 4.56 & 49 & 3 & 400 & 2.85 \\
\hline 5 & 5 & 80 & 4.49 & 50 & 3 & 200 & 2.81 \\
\hline 6 & 5 & 66.66 & 4.43 & 51 & 3 & 133.3 & 2.77 \\
\hline 7 & 4.75 & 400 & 4.52 & 52 & 3 & 100 & 2.73 \\
\hline 8 & 4.75 & 200 & 4.46 & 53 & 3 & 80 & 2.69 \\
\hline 9 & 4.75 & 133.3 & 4.39 & 54 & 3 & 66.66 & 2.66 \\
\hline 10 & 4.75 & 100 & 4.33 & 55 & 2.75 & 400 & 2.62 \\
\hline 11 & 4.75 & 80 & 4.27 & 56 & 2.75 & 200 & 2.58 \\
\hline 12 & 4.75 & 66.66 & 4.20 & 57 & 2.75 & 133.3 & 2.54 \\
\hline 13 & 4.5 & 400 & 4.28 & 58 & 2.75 & 100 & 2.51 \\
\hline 14 & 4.5 & 200 & 4.22 & 59 & 2.75 & 80 & 2.47 \\
\hline 15 & 4.5 & 133.3 & 4.16 & 60 & 2.75 & 66.66 & 2.43 \\
\hline 16 & 4.5 & 100 & 4.10 & 61 & 2.5 & 400 & 2.38 \\
\hline 17 & 4.5 & 80 & 4.04 & 62 & 2.5 & 200 & 2.35 \\
\hline 18 & 4.5 & 66.66 & 3.98 & 63 & 2.5 & 133.3 & 2.31 \\
\hline 19 & 4.25 & 400 & 4.04 & 64 & 2.5 & 100 & 2.28 \\
\hline 20 & 4.25 & 200 & 3.99 & 65 & 2.5 & 80 & 2.25 \\
\hline 21 & 4.25 & 133.3 & 3.93 & 66 & 2.5 & 66.66 & 2.21 \\
\hline 22 & 4.25 & 100 & 3.87 & 67 & 2.25 & 400 & 2.14 \\
\hline 23 & 4.25 & 80 & 3.82 & 68 & 2.25 & 200 & 2.11 \\
\hline 24 & 4.25 & 66.66 & 3.76 & 69 & 2.25 & 133.3 & 2.08 \\
\hline 25 & 4 & 400 & 3.81 & 70 & 2.25 & 100 & 2.05 \\
\hline 26 & 4 & 200 & 3.75 & 71 & 2.25 & 80 & 2.02 \\
\hline 27 & 4 & 133.3 & 3.70 & 72 & 2.25 & 66.66 & 1.99 \\
\hline 28 & 4 & 100 & 3.65 & 73 & 2 & 400 & 1.90 \\
\hline 29 & 4 & 80 & 3.59 & 74 & 2 & 200 & 1.88 \\
\hline 30 & 4 & 66.66 & 3.54 & 75 & 2 & 133.3 & 1.85 \\
\hline 31 & 3.75 & 400 & 3.57 & 76 & 2 & 100 & 1.82 \\
\hline 32 & 3.75 & 200 & 3.52 & 77 & 2 & 80 & 1.80 \\
\hline 33 & 3.75 & 133.3 & 3.47 & 78 & 2 & 66.66 & 1.77 \\
\hline 34 & 3.75 & 100 & 3.42 & 79 & 1.75 & 400 & 1.66 \\
\hline 35 & 3.75 & 80 & 3.37 & 80 & 1.75 & 200 & 1.64 \\
\hline 36 & 3.75 & 66.66 & 3.32 & 81 & 1.75 & 133.3 & 1.62 \\
\hline 37 & 3.5 & 400 & 3.33 & 82 & 1.75 & 100 & 1.60 \\
\hline 38 & 3.5 & 200 & 3.28 & 83 & 1.75 & 80 & 1.57 \\
\hline 39 & 3.5 & 133.3 & 3.24 & 84 & 1.75 & 66.66 & 1.55 \\
\hline 40 & 3.5 & 100 & 3.19 & 85 & 1.5 & 400 & 1.43 \\
\hline 41 & 3.5 & 80 & 3.14 & 86 & 1.5 & 200 & 1.41 \\
\hline 42 & 3.5 & 66.66 & 3.10 & 87 & 1.5 & 133.3 & 1.39 \\
\hline 43 & 3.25 & 400 & 3.09 & 88 & 1.5 & 100 & 1.37 \\
\hline 44 & 3.25 & 200 & 3.05 & 89 & 1.5 & 80 & 1.35 \\
\hline 45 & 3.25 & 133.3 & 3.01 & 90 & 1.5 & 66.66 & 1.33 \\
\hline
\end{tabular}

where:

$\mathrm{N}$ - sample number

$\mathrm{P}$ - adjustable laser power [W]

$\mathrm{f}$ - laser pulse frequency $[\mathrm{kHz}]$

$\mathrm{F}$ - laser radiation fluence $\left[\mathrm{J} / \mathrm{cm}^{2}\right]$ 
Table 2: Results of measurements of geometry of tested samples

\begin{tabular}{|c|c|c|c|c|c|c|c|c|c|}
\hline $\mathrm{N}$ & $\mathrm{h}_{p}[\mu \mathrm{m}]$ & $\mathrm{V}[\mu \mathrm{m}]$ & $\mathrm{h}_{w}[\mu \mathrm{m}]$ & $\Delta \emptyset[\mu \mathrm{m}]$ & $\mathrm{N}$ & $\mathrm{h}_{p}[\mu \mathrm{m}]$ & $\mathrm{V}[\mu \mathrm{m}]$ & $\mathrm{h}_{w}[\mu \mathrm{m}]$ & $\Delta \emptyset[\mu \mathrm{m}]$ \\
\hline 1 & 3.37 & 7039.11 & 1.03 & 1.08 & 46 & 0.83 & 2339.58 & 0.12 & 0.10 \\
\hline 2 & 1.31 & 4691.53 & 0.74 & 0.99 & 47 & 1.03 & 3085.22 & 0.27 & 0.10 \\
\hline 3 & 1.27 & 4659.95 & 0.73 & 0.93 & 48 & 0.82 & 2042.91 & 0.19 & 0.09 \\
\hline 4 & 1.11 & 3842.18 & 0.68 & 0.81 & 49 & 3.61 & 5577.23 & 0.58 & 0.12 \\
\hline 5 & 0.86 & 3338.72 & 0.56 & 0.78 & 50 & 1.82 & 5276.71 & 0.53 & 0.11 \\
\hline 6 & 0.84 & 2052.12 & 0.62 & 0.74 & 51 & 1.61 & 4681.09 & 0.42 & 0.10 \\
\hline 7 & 2.86 & 8878.55 & 2.64 & 1.04 & 52 & 0.85 & 1839.51 & 0.16 & 0.09 \\
\hline 8 & 1.91 & 5191.08 & 1.04 & 0.95 & 53 & 1.01 & 2735.81 & 0.26 & 0.08 \\
\hline 9 & 1.84 & 4874.18 & 0.56 & 0.89 & 54 & 0.97 & 2370.89 & 0.24 & 0.08 \\
\hline 10 & 1.38 & 4638.32 & 0.61 & 0.78 & 55 & 2.70 & 7548.41 & 0.78 & 0.25 \\
\hline 11 & 1.13 & 3984.54 & 0.57 & 0.75 & 56 & 1.87 & 5981.52 & 0.48 & 0.23 \\
\hline 12 & 1.15 & 3641.88 & 0.54 & 0.71 & 57 & 1.12 & 2830.57 & 0.22 & 0.22 \\
\hline 13 & 2.35 & 4840.24 & 1.22 & 0.97 & 58 & 1.07 & 2945.46 & 0.25 & 0.19 \\
\hline 14 & 1.64 & 3594.67 & 0.76 & 0.89 & 59 & 0.67 & 1602.12 & 0.14 & 0.18 \\
\hline 15 & 1.98 & 3771.97 & 0.55 & 0.84 & 60 & 0.82 & 1274.65 & 0.21 & 0.17 \\
\hline 16 & 1.44 & 3794.18 & 0.57 & 0.73 & 61 & 2.19 & 4994.17 & 0.39 & 0.22 \\
\hline 17 & 1.53 & 4292.34 & 0.71 & 0.70 & 62 & 1.64 & 4857.72 & 0.36 & 0.20 \\
\hline 18 & 1.39 & 4076.31 & 0.64 & 0.67 & 63 & 1.03 & 3358.46 & 0.33 & 0.19 \\
\hline 19 & 2.54 & 6003.54 & 0.59 & 0.88 & 64 & 0.92 & 2644.21 & 0.23 & 0.17 \\
\hline 20 & 1.56 & 5085.85 & 0.63 & 0.80 & 65 & 0.85 & 2190.64 & 0.19 & 0.16 \\
\hline 21 & 1.41 & 3424.89 & 0.24 & 0.76 & 66 & 0.71 & 2081.01 & 0.18 & 0.15 \\
\hline 22 & 1.49 & 4525.22 & 0.43 & 0.66 & 67 & 2.56 & 6285.55 & 0.55 & 0.48 \\
\hline 23 & 1.45 & 4181.93 & 0.36 & 0.63 & 68 & 1.06 & 3168.32 & 0.29 & 0.44 \\
\hline 24 & 1.13 & 3576.34 & 0.31 & 0.60 & 69 & 0.91 & 2409.12 & 0.25 & 0.42 \\
\hline 25 & 3.21 & 9787.13 & 0.16 & 0.69 & 70 & 0.81 & 2008.04 & 0.17 & 0.36 \\
\hline 26 & 2.81 & 9734.08 & 0.87 & 0.64 & 71 & 0.88 & 1475.01 & 0.13 & 0.35 \\
\hline 27 & 1.62 & 5949.17 & 0.38 & 0.60 & 72 & 0.51 & 1390.06 & 0.11 & 0.33 \\
\hline 28 & 1.51 & 4912.17 & 0.41 & 0.52 & 73 & 1.28 & 2938.98 & 0.22 & 0.42 \\
\hline 29 & 1.53 & 3369.35 & 0.32 & 0.50 & 74 & 1.26 & 2912.14 & 0.27 & 0.39 \\
\hline 30 & 1.28 & 3742.19 & 0.29 & 0.47 & 75 & 0.94 & 2002.81 & 0.19 & 0.36 \\
\hline 31 & 2.59 & 9614.92 & 0.11 & 0.15 & 76 & 0.89 & 1559.17 & 0.15 & 0.32 \\
\hline 32 & 2.57 & 9524.14 & 0.09 & 0.14 & 77 & 0.88 & 1275.93 & 0.13 & 0.31 \\
\hline 33 & 1.53 & 5751.72 & 0.09 & 0.13 & 78 & 0.57 & 1117.49 & 0.14 & 0.29 \\
\hline 34 & 1.42 & 3579.29 & 0.09 & 0.11 & 79 & 1.69 & 4675.54 & 0.39 & 0.92 \\
\hline 35 & 1.18 & 2912.58 & 0.21 & 0.11 & 80 & 1.23 & 2800.33 & 0.25 & 0.84 \\
\hline 36 & 1.17 & 2405.72 & 0.21 & 0.10 & 81 & 0.87 & 2351.41 & 0.23 & 0.79 \\
\hline 37 & 3.81 & 9024.71 & 1.07 & 0.11 & 82 & 0.67 & 1814.66 & 0.15 & 0.69 \\
\hline 38 & 2.38 & 7792.98 & 0.45 & 0.10 & 83 & 0.74 & 1452.02 & 0.13 & 0.66 \\
\hline 39 & 1.12 & 3611.18 & 0.21 & 0.09 & 84 & 0.41 & 738.32 & 0.06 & 0.63 \\
\hline 40 & 0.85 & 1347.98 & 0.16 & 0.08 & 85 & 1.02 & 1933.32 & 0.17 & 0.81 \\
\hline 41 & 0.85 & 1347.21 & 0.15 & 0.08 & 86 & 0.72 & 1204.47 & 0.11 & 0.74 \\
\hline 42 & 0.79 & 2275.08 & 0.21 & 0.07 & 87 & 0.51 & 1119.04 & 0.01 & 0.69 \\
\hline 43 & 2.73 & 6128.57 & 0.56 & 0.13 & 88 & 0.65 & 933.18 & 0.12 & 0.60 \\
\hline 44 & 0.85 & 3021.65 & 0.19 & 0.12 & 89 & 1.01 & 801.57 & 0.07 & 0.58 \\
\hline 45 & 1.22 & 3102.98 & 0.29 & 0.12 & 90 & 0.42 & 553.71 & 0.05 & 0.55 \\
\hline
\end{tabular}

where:

$\mathrm{h}_{p}$ - microcavity depth obtained $[\mu \mathrm{m}]$

$\mathrm{h}_{w}$ - flash height obtained [ $\left.\mu \mathrm{m}\right]$

$\Delta \emptyset$ - difference between the set and the measured diameter of the microcavity [ $\mu \mathrm{m}]$ 
of the flash and the deviation from the set diameter. Coherent Correlation Interferometry (CCI) based on the collection of interference stripe images and their location during vertical scanning combines vertical scanning with optical interferometry. The limitation of this method when measuring microcavities is the numerical aperture of the lens used, which limits the possibility of measuring on slopes with high inclination. Using a lens with too small aperture for measuring steep slopes will result in areas with nonmeasured points marked as white spots in the surface topography image $[1,5,12]$. Table 2 presents the average values of 3 measurements of the geometry of the tested samples.

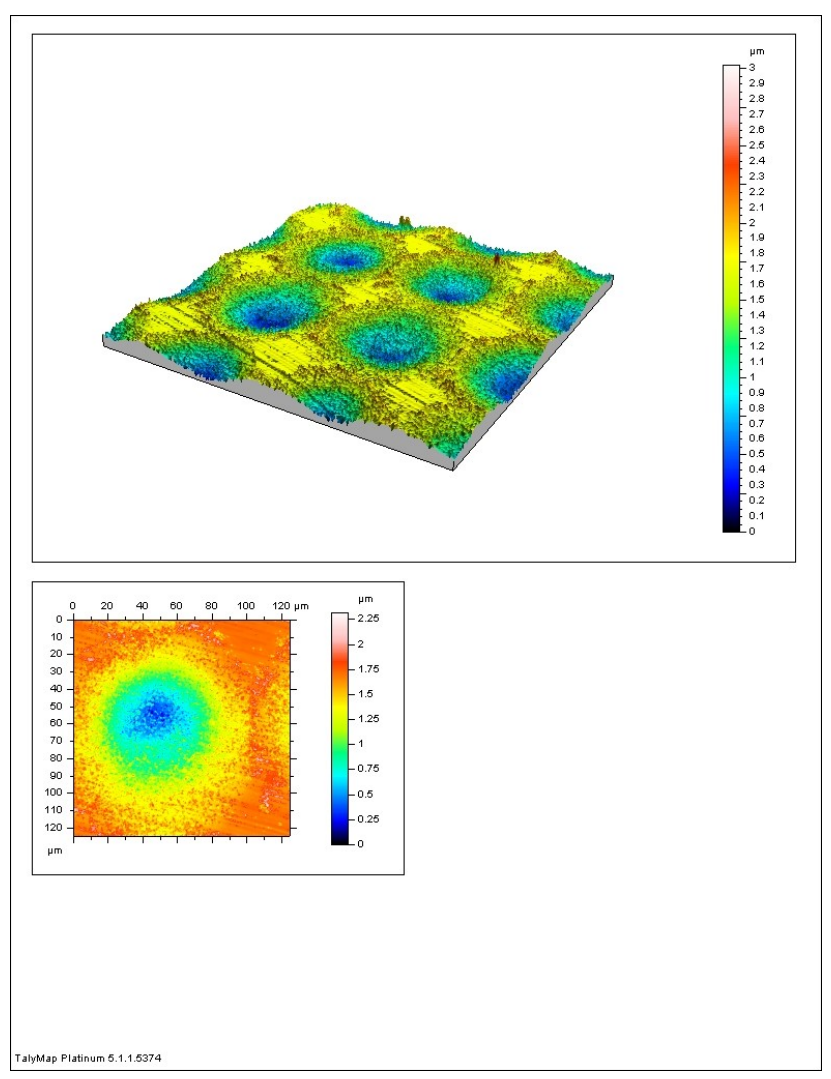

Figure 1: Isometric view of sample obtained with parameters: power $4 \mathrm{~W}$, frequency $133,33 \mathrm{kHz}$, fluency $3,7 \mathrm{~J} / \mathrm{cm}^{2}$

For F-values below $2.62 \mathrm{~J} / \mathrm{cm}^{2}$, there is an insufficient quality of a single texture element due to insufficient energy supplied to the machined surface. Microcavities made at these F-values were inaccurate and their diameter was much smaller than the set diameter. In the case of machining with fluence higher than $3.57 \mathrm{~J} / \mathrm{cm}^{2}$ we observe microcavities of irregular shape resulting from the melting of the material. The diameter of microcavities made at F-values greater than $\mathrm{J} / \mathrm{cm}^{2}$ was greater than the set value.

\section{Analysis of Results}

In order to make a comprehensive assessment of the tested samples, taking into account all the accepted assessment criteria, the multi-criteria optimization method was applied, using the overall desirability function [16-19]. The analysis was performed in the SAS system, version 9.4. In this method, all criteria considered are expressed in a common dimensionless scale. To construct such a scale, it is necessary to specify for each criterion $y^{(i)}$ the range of satisfactory values, indicating inferior value $y_{G}^{(i)}$ and superior value $y_{L}^{(i)}$. If higher values of the criterion are preferable, then $y_{G}^{(i)}<y_{L}^{(i)}$, if smaller values are preferable, then $y_{G}^{(i)}<$ $y_{L}^{(i)}$.

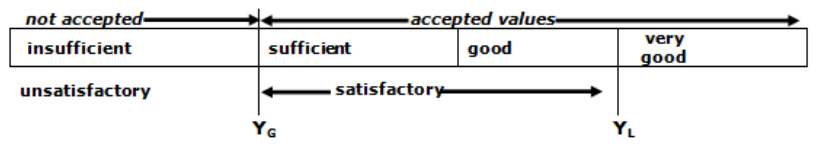

Figure 2: Illustration of the selection of the range of values satisfactory for criterion $Y$ [16-18]

Table 3: Summary of ranges of satisfactory values and weights assigned to particular criteria in two assumed laser texturing applications

\begin{tabular}{|c|c|c|c|c|}
\hline \multirow[t]{2}{*}{ Criteria } & \multicolumn{2}{|c|}{$\begin{array}{c}\text { Ranges of } \\
\text { satisfactory values }\end{array}$} & \multirow{2}{*}{$\begin{array}{c}\text { Weights } \\
1 \text { st } \\
\text { variant }\end{array}$} & \multirow{2}{*}{$\begin{array}{c}\text { Weights } \\
2 \mathrm{nd} \\
\text { variant }\end{array}$} \\
\hline & $\begin{array}{l}\text { inferior } \\
\text { value }\end{array}$ & $\begin{array}{l}\text { superior } \\
\text { value }\end{array}$ & & \\
\hline$h_{p}$ & 1.8 & 2.3 & 0.4 & 0.1 \\
\hline$V$ & 3700 & 4400 & 0.4 & 0.1 \\
\hline$h_{w}$ & 0.6 & 0.2 & 0.1 & 0.3 \\
\hline$\Delta \emptyset$ & 0.8 & 0.5 & 0.1 & 0.5 \\
\hline
\end{tabular}

In addition to ranges of satisfactory values, nonnegative weighting factors reflecting the validity of each criterion are assigned to each criterion. Obviously, the sum of the weights must be equal to 1 . The ranges of satisfactory values and weights for individual criteria should take into account the conditions of the intended use. Partial desirabilities assigned to each criterion $y^{(i)}$ are defined by the following formula:

$$
U_{i}=\exp \left[-\exp \left(-\frac{y^{(i)}-y_{G}^{(i)}}{y_{L}^{(i)}-y_{G}^{(i)}}\right)\right], i=1,2, \ldots m
$$

Desirabilities $U_{i}$ are numbers within the range (0;1), where numbers close to 0 correspond to particularly unfa- 
Table 4: Summary of partial and overall desirabilities for tested samples

\begin{tabular}{|c|c|c|c|c|c|c|}
\hline No. & $\mathrm{Uh}_{p}$ & UV & $U h_{w}$ & $\mathrm{U} \Delta \emptyset$ & $U_{1}$ & $\mathrm{U}_{2}$ \\
\hline 1 & 0.95764 & 0.99156 & 0.0534 & 0.07863 & 0.94966 & 0.35814 \\
\hline 2 & 0.06964 & 0.78461 & 0.24194 & 0.152 & 0.39591 & 0.25183 \\
\hline 3 & 0.05578 & 0.77588 & 0.25057 & 0.21387 & 0.38579 & 0.27289 \\
\hline 4 & 0.01878 & 0.44212 & 0.29482 & 0.35562 & 0.19417 & 0.28488 \\
\hline 5 & 0.00143 & 0.18721 & 0.40461 & 0.39239 & 0.07695 & 0.30579 \\
\hline 6 & 0.00109 & 0.00003 & 0.34949 & 0.44099 & 0.00432 & 0.22751 \\
\hline 7 & 0.88689 & 0.99939 & 0 & 0.10801 & 0.96072 & 0.00186 \\
\hline 8 & 0.4482 & 0.88796 & 0.04958 & 0.1923 & 0.6323 & 0.20352 \\
\hline 9 & 0.39728 & 0.82956 & 0.40461 & 0.25928 & 0.60343 & 0.41767 \\
\hline 10 & 0.09863 & 0.76972 & 0.35868 & 0.39239 & 0.44256 & 0.38904 \\
\hline 11 & 0.02195 & 0.51377 & 0.39545 & 0.42892 & 0.2422 & 0.36573 \\
\hline 12 & 0.02549 & 0.33737 & 0.42286 & 0.47672 & 0.1897 & 0.3777 \\
\hline 13 & 0.71686 & 0.8219 & 0.00899 & 0.17164 & 0.66046 & 0.14109 \\
\hline 14 & 0.2523 & 0.31274 & 0.22496 & 0.25928 & 0.27401 & 0.2464 \\
\hline 15 & 0.49774 & 0.40564 & 0.41375 & 0.31898 & 0.43531 & 0.39316 \\
\hline 16 & 0.12817 & 0.41723 & 0.39545 & 0.45299 & 0.29361 & 0.38588 \\
\hline 17 & 0.17978 & 0.65113 & 0.26806 & 0.48844 & 0.41496 & 0.36535 \\
\hline 18 & 0.10326 & 0.55758 & 0.33115 & 0.52291 & 0.33862 & 0.38691 \\
\hline 19 & 0.79641 & 0.96346 & 0.37708 & 0.27101 & 0.85902 & 0.51482 \\
\hline 20 & 0.19868 & 0.87101 & 0.34031 & 0.36788 & 0.57523 & 0.40917 \\
\hline 21 & 0.11288 & 0.22731 & 0.66593 & 0.41679 & 0.23651 & 0.50214 \\
\hline 22 & 0.15584 & 0.73519 & 0.52008 & 0.53414 & 0.48121 & 0.51442 \\
\hline 23 & 0.13349 & 0.60511 & 0.57764 & 0.56699 & 0.40902 & 0.53487 \\
\hline 24 & 0.02195 & 0.30324 & 0.61611 & 0.59845 & 0.20271 & 0.51525 \\
\hline 25 & 0.94214 & 0.99983 & 0.71686 & 0.50005 & 0.99141 & 0.84926 \\
\hline 26 & 0.87577 & 0.99982 & 0.14029 & 0.55619 & 0.98572 & 0.66226 \\
\hline 27 & 0.23851 & 0.96057 & 0.56161 & 0.59845 & 0.75365 & 0.62655 \\
\hline 28 & 0.16762 & 0.83779 & 0.53693 & 0.67487 & 0.57811 & 0.58791 \\
\hline 29 & 0.17978 & 0.20114 & 0.60861 & 0.6922 & 0.28229 & 0.56121 \\
\hline 30 & 0.05906 & 0.39004 & 0.63084 & 0.71686 & 0.29324 & 0.5838 \\
\hline 31 & 0.81385 & 0.99979 & 0.74546 & 0.89176 & 0.98719 & 0.90142 \\
\hline 32 & 0.80704 & 0.99976 & 0.75621 & 0.89511 & 0.98641 & 0.9031 \\
\hline 33 & 0.17978 & 0.94806 & 0.75621 & 0.89837 & 0.76295 & 0.80818 \\
\hline 34 & 0.11786 & 0.30477 & 0.75621 & 0.9046 & 0.36219 & 0.74748 \\
\hline 35 & 0.03157 & 0.04597 & 0.68578 & 0.9046 & 0.15629 & 0.6769 \\
\hline 36 & 0.02944 & 0.00174 & 0.68578 & 0.90758 & 0.08281 & 0.65955 \\
\hline 37 & 0.98221 & 0.9995 & 0.03924 & 0.9046 & 0.9915 & 0.75413 \\
\hline 38 & 0.73089 & 0.99712 & 0.50294 & 0.90758 & 0.9548 & 0.81523 \\
\hline 39 & 0.02032 & 0.32133 & 0.68578 & 0.91047 & 0.27316 & 0.70444 \\
\hline 40 & 0.00125 & 0 & 0.71686 & 0.91328 & 0.0031 & 0.62175 \\
\hline 41 & 0.00125 & 0 & 0.72278 & 0.91328 & 0.00313 & 0.6254 \\
\hline 42 & 0.00053 & 0.00047 & 0.68578 & 0.916 & 0.02728 & 0.64147 \\
\hline 43 & 0.85584 & 0.96934 & 0.40461 & 0.89837 & 0.91034 & 0.75151 \\
\hline 44 & 0.00125 & 0.07168 & 0.69852 & 0.90153 & 0.10362 & 0.66744 \\
\hline 45 & 0.04117 & 0.09571 & 0.63084 & 0.90153 & 0.19198 & 0.65669 \\
\hline 46 & 0.00095 & 0.00093 & 0.73993 & 0.90758 & 0.03614 & 0.66908 \\
\hline 47 & 0.00942 & 0.09011 & 0.64517 & 0.90758 & 0.14685 & 0.65792 \\
\hline 48 & 0.00083 & 0.00002 & 0.69852 & 0.91047 & 0.01792 & 0.63512 \\
\hline 49 & 0.97357 & 0.93385 & 0.38627 & 0.90153 & 0.93821 & 0.76866 \\
\hline 50 & 0.38259 & 0.9002 & 0.43194 & 0.9046 & 0.73192 & 0.69391 \\
\hline 51 & 0.23171 & 0.78176 & 0.52854 & 0.90758 & 0.60465 & 0.69906 \\
\hline 52 & 0.00125 & 0 & 0.71686 & 0.91047 & 0.01256 & 0.63926 \\
\hline 53 & 0.00779 & 0.01897 & 0.65219 & 0.91328 & 0.09458 & 0.65199 \\
\hline 54 & 0.0052 & 0.00126 & 0.66593 & 0.91328 & 0.05056 & 0.64209 \\
\hline 55 & 0.84764 & 0.99591 & 0.2084 & 0.85225 & 0.95408 & 0.70591 \\
\hline 56 & 0.41922 & 0.96231 & 0.47672 & 0.86108 & 0.81381 & 0.70717 \\
\hline 57 & 0.02032 & 0.03135 & 0.67927 & 0.86531 & 0.11976 & 0.63656 \\
\hline 58 & 0.01349 & 0.05294 & 0.65911 & 0.87731 & 0.12703 & 0.63625 \\
\hline 59 & 0.00007 & 0 & 0.7286 & 0.88108 & 0.00264 & 0.59926 \\
\hline 60 & 0.00083 & 0 & 0.68578 & 0.88474 & 0.0016 & 0.56967 \\
\hline 61 & 0.63229 & 0.85434 & 0.55347 & 0.86531 & 0.7609 & 0.71814 \\
\hline 62 & 0.2523 & 0.82588 & 0.57764 & 0.87342 & 0.6362 & 0.70061 \\
\hline 63 & 0.00942 & 0.19614 & 0.601 & 0.87731 & 0.17968 & 0.62196 \\
\hline 64 & 0.00299 & 0.0109 & 0.67265 & 0.88474 & 0.06513 & 0.62812 \\
\hline 65 & 0.00125 & 0.00018 & 0.69852 & 0.8883 & 0.02488 & 0.6226 \\
\hline 66 & 0.00014 & 0.00004 & 0.70473 & 0.89176 & 0.01261 & 0.61633 \\
\hline 67 & 0.80355 & 0.97543 & 0.41375 & 0.70882 & 0.89557 & 0.66698 \\
\hline 68 & 0.01236 & 0.11797 & 0.63084 & 0.73993 & 0.13394 & 0.55299 \\
\hline 69 & 0.00266 & 0.0018 & 0.65911 & 0.75445 & 0.03206 & 0.53026 \\
\hline 70 & 0.00072 & 0.00001 & 0.71085 & 0.79398 & 0.01108 & 0.55764 \\
\hline 71 & 0.00184 & 0 & 0.73431 & 0.80001 & 0.00336 & 0.55694 \\
\hline 72 & 0 & 0 & 0.74546 & 0.81161 & 0.00035 & 0.54342 \\
\hline 73 & 0.05906 & 0.05152 & 0.67927 & 0.75445 & 0.15318 & 0.59091 \\
\hline 74 & 0.05262 & 0.04588 & 0.64517 & 0.77495 & 0.14361 & 0.57825 \\
\hline 75 & 0.00376 & 0.00001 & 0.69852 & 0.79398 & 0.01673 & 0.55773 \\
\hline 76 & 0.00209 & 0 & 0.72278 & 0.81718 & 0.0047 & 0.56312 \\
\hline 77 & 0.00184 & 0 & 0.73431 & 0.82261 & 0.00184 & 0.5607 \\
\hline 78 & 0.00001 & 0 & 0.7286 & 0.83303 & 0.00015 & 0.53528 \\
\hline 79 & 0.28763 & 0.78022 & 0.55347 & 0.22496 & 0.53925 & 0.46248 \\
\hline 80 & 0.04386 & 0.0269 & 0.65911 & 0.31898 & 0.08633 & 0.42467 \\
\hline 81 & 0.00162 & 0.00104 & 0.67265 & 0.38014 & 0.01605 & 0.40235 \\
\hline 82 & 0.00007 & 0 & 0.72278 & 0.50005 & 0.00194 & 0.43273 \\
\hline 83 & 0.00024 & 0 & 0.73431 & 0.53414 & 0.00078 & 0.43895 \\
\hline 84 & 0 & 0 & 0.77164 & 0.56699 & 0 & 0.42064 \\
\hline 85 & 0.00858 & 0 & 0.71085 & 0.35562 & 0.0099 & 0.4116 \\
\hline 86 & 0.00017 & 0 & 0.74546 & 0.44099 & 0.00019 & 0.40461 \\
\hline 87 & 0 & 0 & 0.7955 & 0.50005 & 0.00004 & 0.44848 \\
\hline 88 & 0.00005 & 0 & 0.73993 & 0.59845 & 0.00004 & 0.43185 \\
\hline 89 & 0.00779 & 0 & 0.76659 & 0.61859 & 0.00033 & 0.48041 \\
\hline 90 & 0 & 0 & 0.77659 & 0.64752 & 0 & 0.44533 \\
\hline
\end{tabular}

$\mathrm{Uh}_{p}$ - partial desirability of $\mathrm{h}_{p}$ attribute

$U \mathrm{~h}_{w}$ - partial desirability of $\mathrm{h}_{\mathrm{w}}$ attribute

$\mathrm{UV}$ - partial desirability of $\mathrm{V}$ attribute

U $\Delta ø$ - partial desirability of $\Delta \varpi$ attribute $U_{1}$

U1 - overall desirability for the 1st variant

U2 - overall desirability for the 2nd variant 
vorable values of $y^{(i)}$ attributeand those close to 1 - to the most favorable ones. When substituting the "inferior" end of the range of satisfactory values, i.e. $y^{(i)}=y_{G}^{(i)}$, to the formula for $U_{i}$, then the expression in the exponent (in round brackets) will take the value 0 . Then $U_{i}=\exp [\exp (0)]=$ 0.368 . In turn, when substituting $y^{(i)}=y_{L}^{(i)}$, which is the "superior" end of the range of satisfactory values, we get the value of 1 in the exponent. Thus, the desirability value is $U_{i}=\exp [\exp (1)]=0.692$. Set of values $y^{(i)}$ considered satisfactory is therefore converted into a range (0.368; 0.692).

The overall desirability $U$ is defined as the weighted exponential mean of the partial desirabilities $U_{i}$.

$$
U=\exp \left[-\exp \sum_{i=1}^{m} w_{i}\left(-\frac{y^{(i)}-y_{G}^{(i)}}{y_{L}^{(i)}-y_{G}^{(i)}}\right)\right]
$$

where $w_{i}$ are the weights assigned to each criterion $y^{(i)}$,

$$
0 \leq w_{i} \leq 1 ; i=1,2, \ldots m ; \sum_{i=1}^{m} w_{i}=1
$$

The overall desirability $U$ takes values from the range $(0,1)$, with higher values of $U$ corresponding to more favorable properties of the tested sample.

By determining the overall desirability value it is possible to assess the tested sample on the basis of the whole set of criteria adopted, taking into account their validity. Optimization utilizing the desirability starts with numerous criteria, while in the last phase the task is reduced to single-criteria optimization. This is due to the fact that the overall desirability is a complex criterion, integrating all the requirements in the form of a single indicator. By determining the overall desirability value for individual samples, it is possible to classify them according to their suitability for the intended use.

The change of weights and ranges of satisfactory values does not require the repetition of tests, but only the repetition of calculations. This way it is made possible to, using the results of a single test cycle, consider multiple potential applications and determine the optimal solution each time.

This multi-criteria optimization method was used to determine solutions for two assumed laser texturing applications:

1st variant. Improvement of tribological properties of friction pairs (increase of hydrodynamic load capacity, reduction of friction resistance).

2nd variant. Surface enhancement for changes in surface energy (gluing, printing, photovoltaic applications).

Table 3 summarizes the ranges of satisfactory values and weights assigned to the criteria in two examples of laser texturing applications. The values in Table 3 are based on literature studies and the author's own experimental research [1, 5, 12, 20-22].

Table 4 presents the calculated partial and overall desirability values in the two assumed applications of laser texturing.

\section{Conclusions}

Research shows that the most important factor influencing the operational behavior of a textured surface is the microgeometry of individual texture elements. Basing on literature studies and the author's own experimental research ranges of satisfactory values and weights assigned to particular criteria in two assumed laser texturing applications were chosen.

Table 4 shows that samples number: $37(U=0.9915)$, 25 ( $\mathrm{U}=0.9914), 31(\mathrm{U}=0.9872), 32(\mathrm{U}=0.9864)$ have the most favorable properties for the first variant of laser texturing application considered. In the case of the second variant of application considered, the samples numbered 32 $(\mathrm{U}=0.9031)$ and $31(\mathrm{U}=0 ., 9014)$ have the highest overall desirability. Table 5 shows the processing parameters for samples of the highest desirability.

Table 5: Processing parameters of samples of the highest desirability

\begin{tabular}{ccccc}
\hline Sample no. & $\begin{array}{c}\mathrm{v} \\
{[\mathrm{mm} / \mathrm{sec}]}\end{array}$ & $\mathrm{P}[\mathrm{W}]$ & $\mathrm{f}[\mathrm{kHz}]$ & $\begin{array}{c}\mathrm{F} \\
{\left[\mathrm{J} / \mathrm{cm}^{2}\right]}\end{array}$ \\
\hline 25 & 100 & 4 & 400 & 3.81 \\
31 & 100 & 3.75 & 400 & 3.57 \\
32 & 100 & 3.75 & 200 & 3.52 \\
37 & 100 & 3.5 & 400 & 3.33 \\
\hline
\end{tabular}

Since the fluence during the production of sample no. 25 amounted to 3.81, this set of processing parameters should be rejected because they do not fall within the range in which cold laser ablation and a satisfactory regularity of the microcavity according to Table 2 is achieved. Identical desirabilities and geometry parameters of samples can be obtained with any laser micromachine while maintaining laser radiation fluence at the appropriate level.

Application of statistical functions during the selection of machining parameters will allow to develop the application of laser texturing on an industrial scale and bet- 
ter use of the benefits that it brings in the considered applications.

\section{References}

[1] Sęk P. Wytwarzanie i własności powierzchni z teksturą, Wydawnictwo Politechniki Świętokrzyskiej, Kielce 2014

[2] Vemak V, Dahotre N. Lasers in Surface Engineering. Surface Engineering Series. ASM International. Materials Park. OH USA. 1998

[3] Gregson V. Laser Material Processing. Holland Publishing Company. 1984

[4] Ion JC. Laser Processing of Engineering Materials: Principles, Procedure and Industrial Applications. Elsevier ButterworthHeinemann. ISBN: 9780750660792

[5] Antoszewski B, Sęk P. Laser surface texturing - chosen problems. Proc. SPIE 8703, Laser Technology 2012: Applications of Lasers, 87030H, DOI: https://doi.org/10.1117/12.2015240

[6] Otmianowski T, Antoszewski B, Żórawski W. Local laser treatment of tribological plasma sprayed coatings. Proc. of $15^{\text {th }}$ International Thermal Spray Conference, 25-29 May, Nice, France, 1998, 1333-1336.

[7] Góral A, Lityńska-Dobrzyńska L, Żórawski W, Berent K, WojewodaBudka J. Microstructure of $\mathrm{Al}_{2} \mathrm{O}_{3}-13 \mathrm{TiO}_{2}$ coatings deposited from nanoparticles by plasma spraying. Arch. Metall. Mater. 58(2013)2. 335-339, DOI: 10.2478/v10172-012-0194-1

[8] Żórawski W, Makrenek M, Góral A. Mechanical properties and corrosion resistance of HVOF sprayed coatings using nanostructured carbide powders. Arch. Metall. Mater.,Vol.61. (2016), No 4, 1839-1846. DOI: 10.1515/amm-2016-0297

[9] Chichkov BN, Momma C, Nolte S, von Alvensleben F, Tünnermann A. Femtosecond, picosecond and nanosecond laser ablation of solid. Appl Phys A-Mater 63, 109-115, DOI: https://doi.org/10.1007/BF01567637

[10] Chrisey DB, Hubler GK. Pulsed Laser Deposition of Thin Films. Wiley-Interscience. New York. 1994

[11] Żenkiewicz M. Adhezja i modyfikowanie warstwy wierzchniej tworzyw wielocząsteczkowych. WNT Warszawa 2000. ISBN 83 204-2547-6
[12] Radek N, Bartkowiak K. Laser treatment of electro-spark coatings deposited in the carbon steel substrate with using nanostructured WC-Cu electrodes. Phys. Procedia. 2012, 39, 295-301, DOI: https://doi.org/10.1016/j.phpro.2012.10.041

[13] Scendo M, Trela J, Radek N. Influence of laser power on the corrosive resistance of WC-Cu coating. Surf. Coat. Technol. 2014, 259, 401-407, DOI: https://doi.org/10.1016/j.surfcoat.2014.10.062

[14] Radek N, Bartkowiak K. Laser treatment of Cu-Mo electro-spark deposited coatings. Phys. Procedia. 2011, 12, 499-505, DOI: https://doi.org/10.1016/j.phpro.2011.03.061

[15] Pietraszek J, Radek N, Bartkowiak K. Advanced statistical refinement of surface layer's discretization in the case of electro-spark deposited carbide-ceramic coatings modified by a laser beam. Solid State Phenom. 197 (2013), 198-202. https://doi.org/10.4028/www.scientific.net/SSP.197.198

[16] Grabiec A, Piasta Z. Analysis of superplasticizer influence on cement paste with multicriteria statistical optimization. Archives of Civil Engineering XLII, 1996

[17] Harrington EC. The desirability function. Industrial Quality Control. 21. 494-498, 1965

[18] Pettersson U, Jacobson S. Influence of surface texture on boundary lubricated sliding contacts. Tribol. Int. 36. 857-864, DOI: https://doi.org/10.1016/S0301-679X(03)00104-X

[19] Piasta Z, Lenarcik A. Applications of statistical multi-criteria optimisation in design of concretes, in Optimization Methods for Material Design of Cement-based Composites. E \& FN Spon. London, 1998

[20] Nosal S. Tribologia. Wprowadzenie do zagadnień tarcia, zużywania i smarowania. Oficyna Wydawnicza Politechniki Opolskiej. 2011, ISBN 978-83-7775-146-6

[21] Radek N, Bartkowiak K. Laser treatment of electro-spark coatings deposited in the carbon steel substrate with using nanostructured WC-Cu electrodes. Phys. Procedia. 2012, 39, 295-301, DOI: https://doi.org/10.1016/j.phpro.2012.10.041

[22] Owens DK, Wendt RC. Estimation of the surface free energy of polymers. J. Appl. Polym. Sci. vol. 13. 1741-1747, DOI: https://doi.org/10.1002/app.1969.070130815 